\title{
Nogo-C regulates cardiomyocyte apoptosis during mouse myocardial infarction
}

\author{
Shi Jia ${ }^{1}$, Xue Qiao ${ }^{1}$, Jingjing Ye ${ }^{1}$, Xuan Fang ${ }^{1}$, Chunling $\mathrm{Xu}^{1}$, Yangpo Cao ${ }^{1}$ and Ming Zheng ${ }^{\star, 1}$
}

Myocardial infarction is caused by insufficient coronary blood supply, which leads to myocardial damage and eventually the heart failure. Molecular mechanisms associated with the loss of cardiomyocytes during myocardial infarction (MI) and ischemia-related cardiac diseases are not yet fully understood. Nogo-C is an endoplasmic reticulum protein ubiquitously expressed in tissues including in the heart, however, the cardiac function of Nogo-C is still unknown. In the present study, we found that Nogo-C was upregulated in mouse hearts after $\mathrm{MI}$, and hypoxic treatments also increased Nogo-C protein level in cardiomyocytes. Adenovirus mediated overexpression of Nogo-C led to cardiomyocyte apoptosis, whereas knockdown of Nogo-c by shRNA protected cardiomyocytes from hypoxia-induced cell apoptosis. Importantly, Nogo-C knockout mice displayed improved cardiac function, smaller infarct area, and less apoptotic cells after MI. Moreover, we found that miR-182 negatively regulated Nogo-C expression and was downregulated during MI, expressing miR-182 in cardiomyocytes protected hypoxia- and Nogo-C-mediated cell apoptosis. Our results indicate that increased cardiac Nogo-C expression is both sufficient and necessary for ischemia-induced cardiomyocyte apoptosis and cardiac dysfunction, suggesting that deregulation of Nogo-C by miRNA may be a potential therapeutic target for ischemia-related heart diseases.

Cell Death and Disease (2016) 7, e2432; doi:10.1038/cddis.2016.331; published online 20 October 2016

Myocardial infarction (MI) causes myocardial damage and eventually leads to heart failure, the leading cause of death worldwide. ${ }^{1,2} \mathrm{Ml}$ is characterized by insufficient coronary blood supply, and the prolonged ischemia during MI causes loss of cardiomyocytes due to apoptosis and necrosis. ${ }^{3}$ Although various signaling pathways involved in cardiomyocyte apoptosis including reactive oxygen species (ROS), protein kinase $\mathrm{C}, \mathrm{MAPK}$, and nuclear factor $\mathrm{kB}$, have been reported, ${ }^{4-7}$ the molecular mechanisms associated with $\mathrm{MI}$ and ischemia-related cardiac diseases are not yet fully understood.

Neurite outgrowth inhibitor proteins (Nogo) belong to reticulon (RTN) protein family, which is characterized by the endoplasmic reticulum targeting motif at the carboxy terminal. $^{8}$ Nogo gene encodes three splicing isoforms, Nogo-A, Nogo-B and Nogo-C. Nogo-A is a membrane protein expressed mainly in central nervous system such as oligodendrocytes and neurons, serving as a growth inhibitory factor and restricting axon re-extension. ${ }^{9,10}$ Recently, Nogo-A has also been found as an important negative regulator of developmental angiogenesis in central nervous system. ${ }^{11}$ Nogo-B is a shorter isoform than Nogo-A, expressed ubiquitously in the body. ${ }^{12}$ In peripheral nervous system, Nogo-B is expressed in Schwann cells and the interaction of Nogo-B with its receptor on neurons mediates axonal branching, indicating a role in the excessive axonal sprouting after peripheral nerve injury. ${ }^{13}$ In peripheral blood vessels,
Nogo-B regulates endothelial cell migration thus mediating vascular remodeling after lesions. ${ }^{14}$ And in pulmonary arterial hypertension patients, Nogo-B is increased and causes pulmonary arterial smooth muscle cell over-proliferation due to suppression of cell apoptosis. ${ }^{15}$ Despite these important functions from in vitro studies, either Nogo-A or Nogo-B knockout mouse displays relatively normal phenotypes. ${ }^{16}$ Nogo-C is the shortest protein in Nogo family, and expressed in many tissues, including liver, neuron, vascular smooth muscle cells, skeletal muscles, and heart. ${ }^{17}$ In transgenic mice expressing Nogo-C in Schwann cells, sciatic nerve injury causes delayed axonal regeneration and decreased recovery of motor function. ${ }^{18}$ In hepatic carcinoma, the protein level of Nogo-C is negatively correlated with tumor size and prognosis. ${ }^{19,20}$ However, the role of Nogo-C protein in cardiac pathogenesis has not been investigated.

As Nogo proteins have been reported to mediate multiple cell apoptosis, ${ }^{21-23}$ and apoptosis is a common feature of cardiac infarction, we then hypothesize that Nogo- $\mathrm{C}$ may have a role in mediating cardiomyocyte apoptosis during cardiac infarction. In the present study, using combination of in vitro and in vivo approaches, we investigated the role of Nogo-C in $\mathrm{Ml}$ and ischemic cardiomyocytes. We found upregulated protein level of Nogo-C in Ml heart tissues and hypoxic cardiomyocytes. Nogo-C deletion preserved cardiac functions after Ml. Our study provides in vivo evidence, for the first time, that Nogo- $\mathrm{C}$ has a pivotal role in regulating

\footnotetext{
${ }^{1}$ Department of Physiology and Pathophysiology, Health Science Center, Peking University, Beijing 100191, China

*Corresponding author: M Zheng, Department of Physiology and Pathophysiology, Health Science Center, Peking University, Xueyuan Road 38, Beijing 100191, China. Tel: +86 108280 2403; Fax: 86108280 2403; E-mail: zhengm @bjmu.edu.cn

Abbreviations: MI, myocardial infarction; ROS, reactive oxygen species; 3'UTR, 3' untranslated regions; TUNEL, terminal deoxyribonucleotidyl transferase (TDT)mediated dUTP-digoxigenin nick end labeling; AV, annexin V; TTC, triphenyltetrazolium chloride; IR, infarct size; LV, left ventricule; LAD, lactate dehydrogenase; shRNA, short hairpin RNA; miRNA, microRNA; LVID, left ventricular internal diameter; LVPW, left ventricular posterior wall thickness; EF, ejection fraction; FAC, fractional area change; HW/BW, heart weight to body weight ratio; HW/TL, heart weight to tibia length ratio; HE, hematoxylin/eosin

Received 03.7.16; revised 17.9.16; accepted 20.9.16; Edited by J Chipuk
} 
a

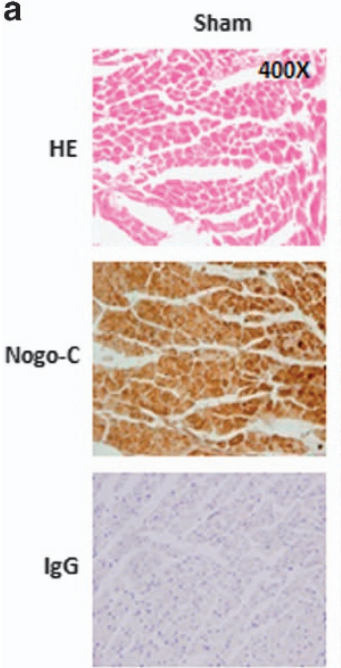

C
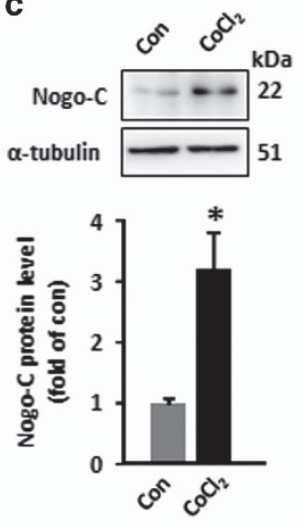

b
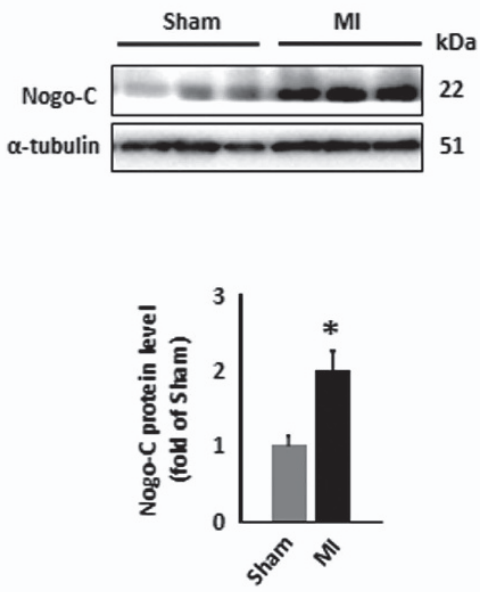

d
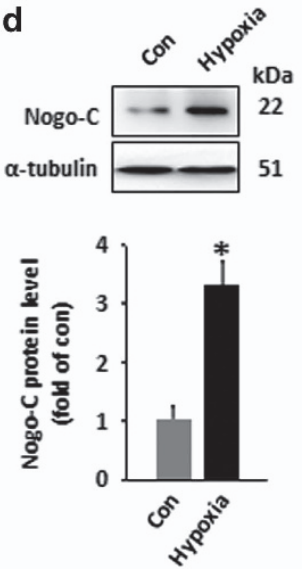

Figure 1 Nogo-C is upregulated in MI hearts and hypoxic cardiomyocytes. (a) Hematoxylin/eosin staining and Nogo-C immunohistochemical staining of sham and Ml mouse hearts, IgG staining as control. (b) Western blot and average data showing Nogo-C protein levels in sham and Ml mouse hearts $24 \mathrm{~h}$ after operation. $n=6-7 \mathrm{mice}$. (c) and (d) Nogo-C protein levels in rat neonatal cardiomyocytes treated with $\mathrm{CoCl}_{2}(600 \mu \mathrm{M})$ for $24 \mathrm{~h}(\mathbf{c})$ or with hypoxia incubation $\left(1 \% \mathrm{O}_{2}\right)$ for $12 \mathrm{~h}(\mathrm{~d})$. $n=6$ independent experiments. ${ }^{*} P<0.05$ versus sham group or control cells

cardiac function and may serve as a potential therapeutic target for clinical MI treatment.

\section{Results}

Nogo-C expression was upregulated in MI heart and hypoxic cardiomyocytes. Nogo-C expressed ubiquitously in mouse tissues including the heart as indicated by its expression pattern (data not shown). To understand the pathological role of Nogo-C in the heart, we checked the expression level in MI. Immunohistochemical staining by Nogo-C antibody showed that Nogo- $\mathrm{C}$ was upregulated in the border zone of infarct area $24 \mathrm{~h}$ after LAD (Figure 1a). Western blot result confirmed the increased Nogo-C protein level in the border zone of MI, as indicated by the 2.3-fold increase in MI mouse heart than in sham control (Figure 1b). We then tested if hypoxia promoted Nogo-C protein level in cultured neonatal cardiomyocytes by two approaches. First, we treated cardiomyocytes with $\mathrm{CoCl}_{2}$, a compound commonly used as a hypoxic inducer due to its role in inducing and stabilizing hypoxia inducible factor $1 a$ (HIF-1a). $\mathrm{CoCl}_{2}(600 \mu \mathrm{M}$ for $24 \mathrm{~h})$ caused increased Nogo-C protein level to 3.2-fold of that in vehicle control cells (Figure 1c). Then, we cultured cardiomyocytes in hypoxic incubator with gas containing $94 \% \mathrm{~N}_{2}, 1 \% \mathrm{O}_{2}$ and $5 \% \mathrm{CO}_{2}$ for $12 \mathrm{~h}$, and found that hypoxia treatment also increased Nogo-C to 3.3-fold comparing with cardiomyocytes cultured in normoxia condition (Figure 1d). Together, these results showed that Nogo-C protein was changed during hypoxia or $\mathrm{Ml}$, suggesting that Nogo-C may have a role in the pathogenesis of hypoxia-related cardiac diseases.

Nogo-C induced cardiomyocyte apoptosis. We next investigated if alteration of Nogo-C protein level mediated cardiomyocyte function. Transfection of rat neonatal cardiomyoyctes with adenovirus containing Nogo-C cDNA (Ad-Nogo-C) caused increased cellular Nogo-C protein level (Figure 2a). Overexpression of Nogo-C increased 
cardiomyocyte apoptosis, as indicated by flow cytometry assay, with a LR (LR quadrant indicates the percentage of early apoptotic cells to Alexa 488 stained cells) of $17.53 \pm 3.085 \%$ in Ad-Nogo-C expressing cells and $6.4 \pm 1.112 \%$ in vector control cells, and a UR (UR quadrant indicates the percentage of late apoptotic cells to Alexa 488 and propidium iodide-stained cells) of $9.982 \pm 1.045 \%$ in AdNogo- $C$ cells and $7.373 \pm 1.134 \%$ in control (Figure $2 b$ ). The apoptotic effect of Nogo-C on cardiomyocytes was also confirmed by TUNEL staining, with a 2.2-fold increased apoptotic rate in adeno-Nogo-C cells over that in control cells (Figure 2c). Thus, our results suggest that Nogo-C is sufficient to induce cardiomyocyte apoptosis.

We then investigated if decreased Nogo-C protein level could protect cardiomyocytes from apoptotic stimuli by infecting cardiomyocyte with adenovirus containing Nogo-C short hairpin RNA (shRNA) (Ad-sh-Nogo-C) to knockdown Nogo-C protein (Figure 2d). Flow cytometry assay showed that Nogo-C knockdown protected cardiomyocyte apoptosis induced by $\mathrm{CoCl}_{2}$, from $12.61 \pm 3.444 \%$ to $6.103 \pm 1.166 \%$ (LR) and $13.21 \pm 2.472 \%$ to $7.315 \pm 1.637 \%$ (UR) (Figure $2 e$ ). Similarly, TUNEL staining showed that knockdown of Nogo-C decreased $\mathrm{CoCl}_{2}$ induced cardiomyocyte apoptosis from $25.8 \%$ to $13.3 \%$ (Figure 2f). Altogether, our results showed that Nogo-C is both sufficient and necessary for cardiomyocyte apoptosis.

Knockout of Nogo-C protected mice from Ml-induced cardiac dysfunction. To understand the in vivo function of Nogo- $\mathrm{C}$, we generated Nogo- $\mathrm{C}^{-1-}$ mice by TALEN technique to delete the Nogo-C specific exon 1c (Figures $3 a$ and b). Basically, the ratios of heart weight to body weight and heart weight to tibia length in Nogo- $\mathrm{C}^{-l-}$ mice are similar with that in control mice at age of 8 -week old (Figure 3c). Also, left ventricular internal diameter (LVID)/left ventricular posterior wall thickness (LVPW) showed no difference between Nogo$\mathrm{C}^{-1-}$ and control mice (Figure $3 \mathrm{~d}$ ). Cardiomyocyte area by hematoxylin/eosin staining and ultra-structure by tansmission electron microscope are all similar in hearts of Nogo- $\mathrm{C}^{-/-}$and control mice (Figures $3 e$ and f). Moreover, Nogo-C knockout did not alter cardiac function as revealed by echocardiography (Table 1) and electrocardiograph assay (Figure $3 \mathrm{~g}$ ). These data suggest that deletion of Nogo-C does not affect heart morphology and function at basal conditions.

We then investigated the possible effect of Nogo-C deficiency on cardiac function during MI. Although echocardiography analysis showed largely decreased ejection fraction (EF) and fractional area change (FAC) in control mice after MI (EF: $67.26 \pm 3.148 \%$ versus $23.51 \pm 3.694 \%$, FAC: $50.9 \pm 2.992 \%$ versus $15.44 \pm 2.338 \%$, before and after $\mathrm{MI}$ ), Nogo-C knockout significantly improved cardiac function after $\mathrm{Ml}$ to $44.61 \pm 4.164 \%$ (EF) and $30.49 \pm 2.77 \%$ (FAC) (Figure $4 a$ ). The infarct size (IR) of Nogo- $\mathrm{C}^{-/-}$mouse hearts also decreased as comparing with wildtype littermates after $\mathrm{Ml}$ (Figure 4b). Lactate dehydrogenase (LDH) levels in Nogo- $\mathrm{C}^{-/-}$mouse serum after $\mathrm{Ml}$ was reserved as comparing with the increased levels in control littermates after MI $\left(1004 \pm 76.79\right.$ in Nogo- $C^{-1-}$ mouse versus $1740 \pm 289.9$ in control mouse after $\mathrm{MI}$ ) (Figure 4c), indicating that knockout of Nogo-C has a protective effect on Ml-induced heart injury. We further examined cardiomyocyte apoptosis by TUNEL staining after $\mathrm{MI}$, and found that Nogo-C knockout protected cardiomyocyte apoptosis, from $10.64 \pm 2.255 \%$ in control hearts after $\mathrm{Ml}$ to $2.907 \pm 1.163 \%$ in Nogo- $\mathrm{C}^{-/-}$hearts (Figure $4 \mathrm{~d}$ ). Collectively, these data support the protective role of Nogo-C knockout on Ml-induced heart damage.

MiR-182 negatively regulated Nogo-C expression during MI. MiRNAs have a critical role in cardiac pathological processes. In this study we tried to identify the possible miRNA that regulates Nogo-C gene expression during $\mathrm{MI}$. In silico analysis predicted that miR-182 targets the $3^{\prime}$ untranslated region ( $3^{\prime}$ UTR) of Nogo-C (Figure 5a). Importantly, miR-182 expression was significantly decreased in the heart during $\mathrm{Ml}$ (Figure $5 \mathrm{~b}$ ) or in $\mathrm{CoCl}_{2}$-treated cardiomyocytes (Figure 5c). Overexpression of miR-182 dramatically reduced Nogo-C protein expression in cardiomyocytes (Figures 5d and e). In addition, miR-182 decreased Nogo-C 3'-UTR luciferase reporter activity (Figure 5f), further confirming that Nogo-C is a target gene of miRNA-182.

To test if miR-182 had a role in Nogo-C-induced cardiomyocyte apoptosis, cardiomyocytes were co-transfected with miR-182 and Ad-Nogo-C, or transfected with miR-182 in the presence of $\mathrm{CoCl}_{2}$. Although overexpression of Nogo-C or treatment of $\mathrm{CoCl}_{2}$ induced cardiomyocyte apoptosis, the overexpression of miR-182 protected cells from either Nogo-C or $\mathrm{CoCl}_{2}$-induced apoptosis, as assayed by flow cytometry (Figure $5 \mathrm{~g}$ ). Together, our results indicate that miR-182 has an important role in hypoxia-related cardiac diseases through negatively regulating Nogo-C expression.

\section{Discussion}

Nogo family proteins are profoundly involved in multiple cellular processes. In addition to their well studied functions in nervous system, our present study found that Nogo-C is a determinant player in ischemia-related cardiomyocyte apoptosis during MI. There are several lines supporting our notions. First, Nogo-C protein is increased in $\mathrm{MI}$ heart and in hypoxic stimuli-induced apoptotic cardiomyocytes. Second, overexpression of Nogo-C per se is sufficient to induce cardiomyocyte apoptosis, whereas knockdown of Nogo-C prevents hypoxia-induced apoptosis. Third, in vivo knockout of Nogo-C (Nogo- $\mathrm{C}^{-/-}$) preserved cardiac function and protected cardiomyocytes against apoptosis after MI. Finally, we identified Nogo-C is a target gene of miR-182, which negatively regulated Nogo- $\mathrm{C}$ and is downregulated during MI.

Apoptosis is an important feature leading to the cardiac dysfunction after MI and ischemic heart diseases. ${ }^{24,25}$ Thus, investigation of the molecular mechanisms underlying apoptosis is helpful for better understanding $\mathrm{MI}$, and even for providing therapeutic targets for treatment of MI. In our current study, we found that both mRNA and protein levels of Nogo-C were upregulated in $\mathrm{MI}$ hearts, and the Nogo-C protein mainly increased in border area of infarct zone where most apoptotic cells enriched, suggesting a possible role of Nogo-C in the pathogenic process of cardiomyocyte apoptosis. Indeed, overexpression of Nogo-C in cardiomyocytes caused cell apoptosis, and knockdown of Nogo-C inhibited 
a

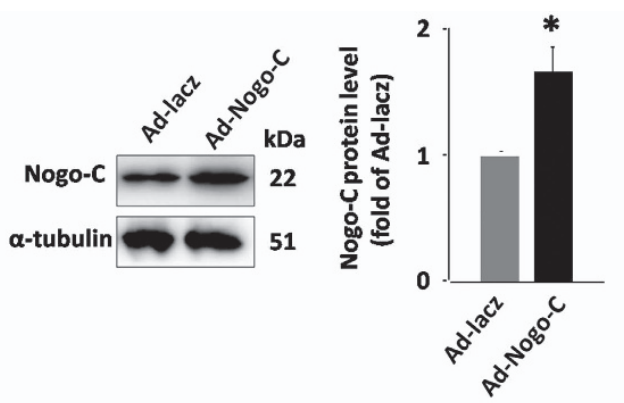

c

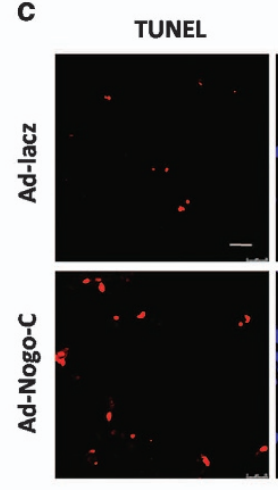

DAPI

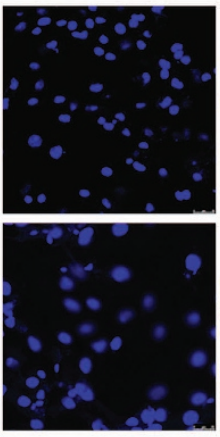

b
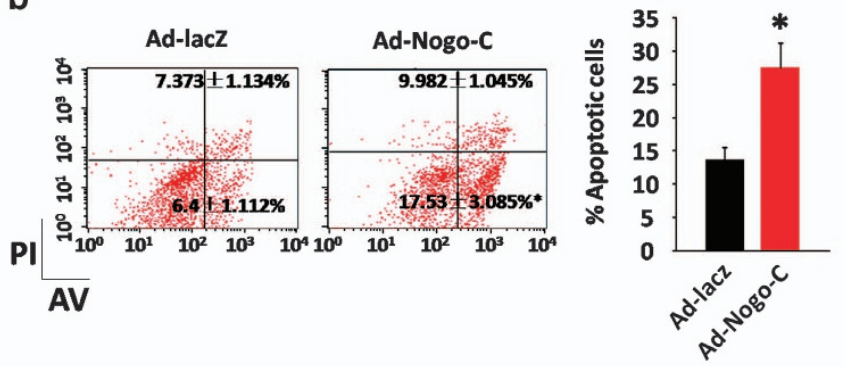

d
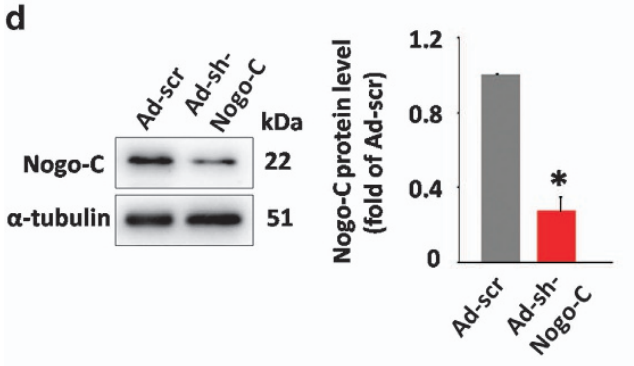

e
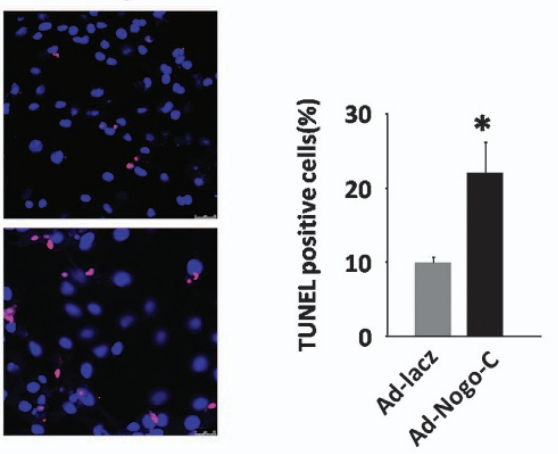

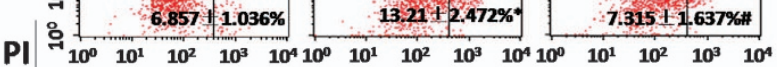

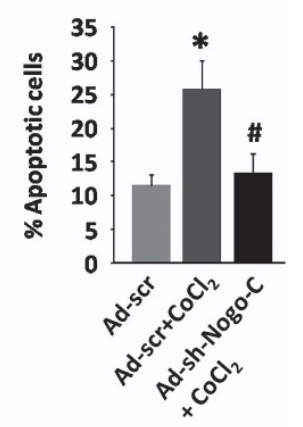

f
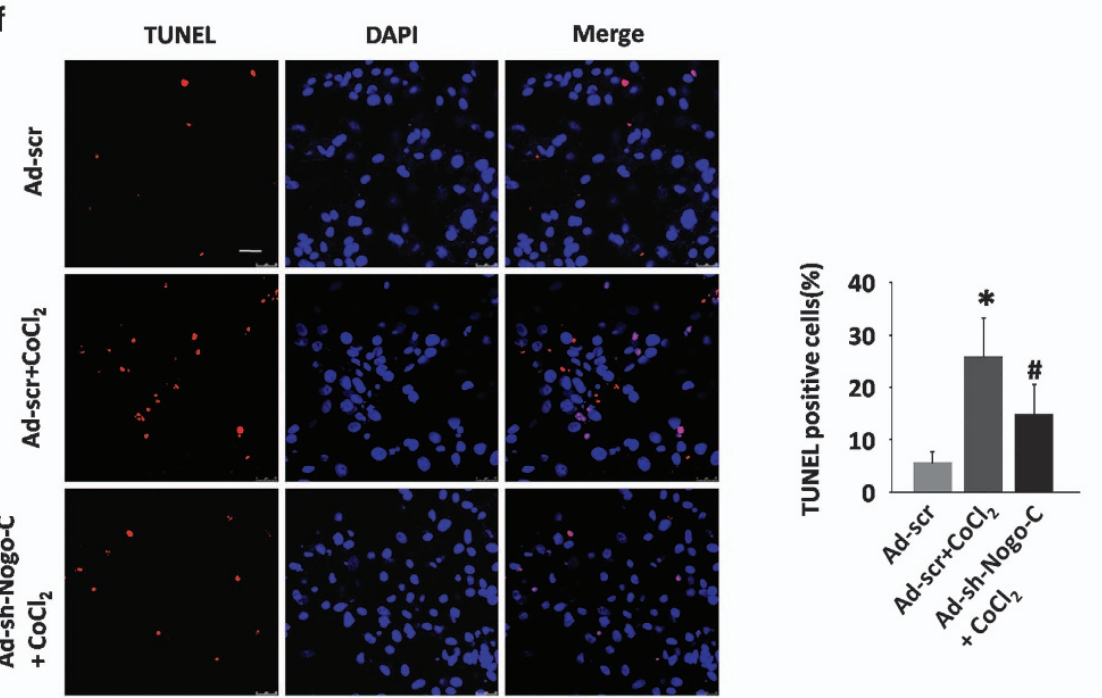

hypoxia-induced cardiomyocyte apoptosis. Our findings that Nogo- $C$ regulates cell apoptosis in the heart are in general agreement with findings of Nogo-A, one of the splicing isoforms of Nogo-C, in cardiomyocytes. ${ }^{23}$ Although highly expressed in central nervous system, Nogo-A has also been reported to be upregulated in human hearts of dilated cardiomyopathy and rat neonatal cardiomyocytes subjected to hypoxia/reperfusion, and knockdown of Nogo-A inhibited 
Figure 2 Nogo-C regulates cardiomyocyte apoptosis. (a) Western blot showing the Nogo-C protein level in rat neonatal cardiomyocytes transfected with Ad-Nogo-C at 50 $\mathrm{MOI}$ or Ad-laz for 48 h. $n=3$ independent experiments. (b) Flow cytometry analysis and (c) TUNEL staining showing apoptotic rates of rat neonatal cardiomyocyte transfected with Ad-Nogo-C or Ad-laz. $n=3$ independent experiments for flow cytometry analysis; and $n=6$ independent experiments for TUNEL staining. Scale bar $=25 \mu$ m. (d) Western blot showing the Nogo-C protein level in rat neonatal cardiomyocytes transfected with Ad-sh-Nogo-C at $50 \mathrm{MOI}$ or Ad-scramble for $48 \mathrm{~h}$. $n=3$ independent experiments. (e) Flow cytometry analysis and (f) TUNEL staining showing apoptotic rates of rat neonatal cardiomyocytes infected with Ad-sh-Nogo-C or $\mathrm{Ad}_{-}-\mathrm{scramble}_{\mathrm{m}}$ in response to $\mathrm{CoCl}_{2}$ stimulation $(600 \mu \mathrm{M}) . n=6$ independent experiments. Scale bar $=25 \mu \mathrm{m} .{ }^{*} P<0.05$ versus scramble control cells. ${ }^{\#} P<0.05$ versus scramble+CoCl ${ }_{2}$ cells

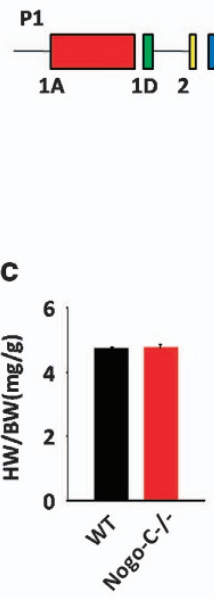

e
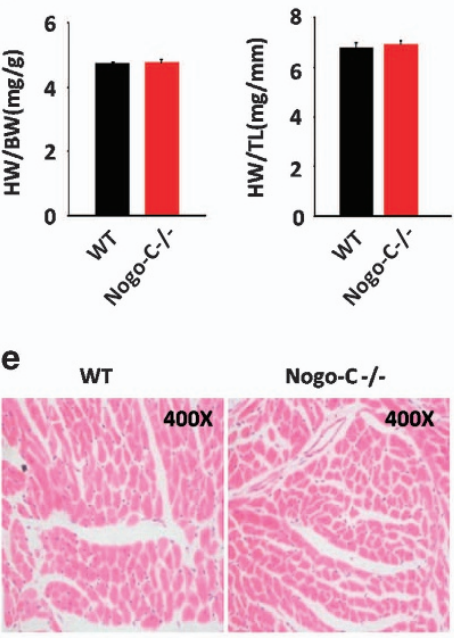

P2

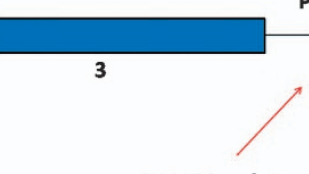

TALEN pair1
1C b

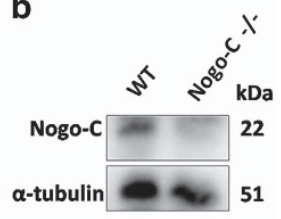

d

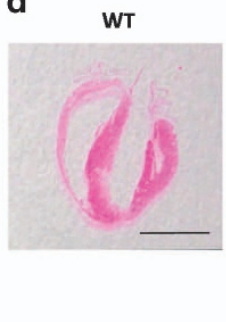

f

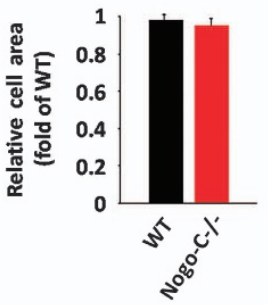

Nogo-C- -

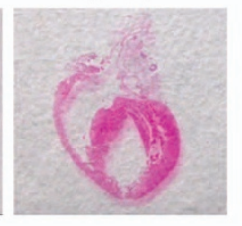

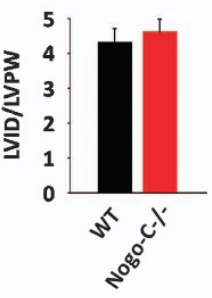

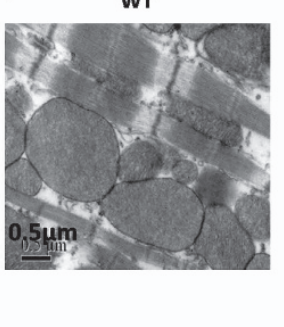

Nogo-C - $/-$

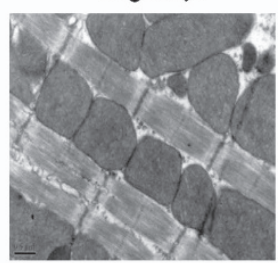

g
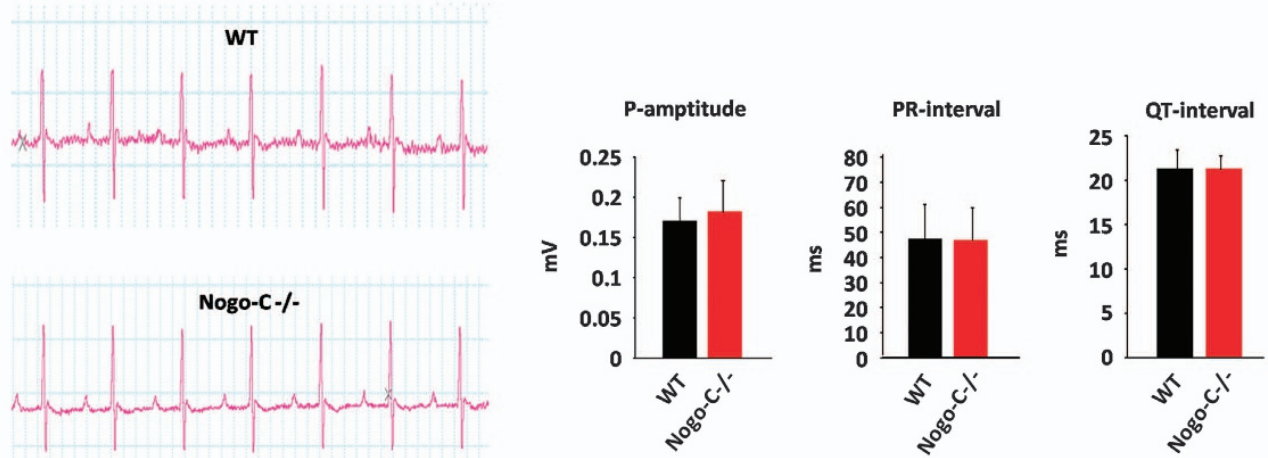

Figure 3 Generation and characterization of Nogo-C Knockout mouse. (a) Schematic diagram of Nogo- $\mathrm{C}^{-1-}$ mouse construction by TALEN technique. (b) Western blot showing the Nogo-C protein level in hearts from wildtype and Nogo- $\mathrm{C}^{-1-}$ mice. (c) Heart weight to body weight ratio (left) and Heart weight to tibia length ratio (HW/TL) (right) of 8-week male WT and Nogo- $C^{-1}$ mice. $n=11$ mice. (d) Hematoxylin and eosin staining showing the heart chambers of 8-week male WT and Nogo- $C^{-1-}$ mice (left), and the diastole LVID to LVPW ratio (right). Scale bar $=0.5 \mathrm{~cm} . n=6$. (e) Cross section image of 8-week male WTand Nogo- $\mathrm{C}^{-1}-$ mouse hearts by hematoxylin and eosin staining (left) and the average data (right). WT, $n=70$ cells (from 4 mice); Nogo- $C^{-\prime-}, n=63$ cells (from 5 mice). Magnifying power: 400X. (f) Representative TEM images of 8-week male WT and Nogo- $\mathrm{C}^{-1-}$ mouse hearts. Scale bar $=0.5 \mu \mathrm{m}$. (g) Electrocardiograph images of 8-week male WTand Nogo- $\mathrm{C}^{-1}$ - mice (left) and statistic data for P-amptitude, PR-interval, and QT-interval (right). $n=4-5$ mice 
Table 1 Echocardiographic assay of cardiac function in wildtype control and Nogo-C knockout mice

\begin{tabular}{lccc}
\hline \multirow{2}{*}{$\begin{array}{l}\text { Echocardiographic } \\
\text { parameter }\end{array}$} & WT $(n=7)$ & & \multirow{2}{*}{ Nogo-C $^{-1-}(n=6)$} \\
\cline { 2 - 2 } & Mean \pm S.E.M. & & Mean \pm S.E.M. \\
\hline LVID-d $(\mathrm{mm})$ & $3.221 \pm 0.0649$ & & $3.417 \pm 0.1072$ \\
LVPW-d $(\mathrm{mm})$ & $0.7886 \pm 0.07595$ & & $0.7633 \pm 0.06647$ \\
LVID-s $(\mathrm{mm})$ & $2.119 \pm 0.107$ & & $2.287 \pm 0.08686$ \\
LVPW-s $(\mathrm{mm})$ & $1.169 \pm 0.09277$ & & $1.13 \pm 0.07014$ \\
EF(\%) & $64.18 \pm 3.544$ & & $62.43 \pm 2.244$ \\
FS(\%) & $34.38 \pm 2.643$ & & $33 \pm 1.575$ \\
& & &
\end{tabular}

Abbreviations: LVID-d, left ventricular internal diameter at end-diastole; LVPW$\mathrm{d}$, left ventricular posterior wall thickness at end-diastole; LVID-s, left ventricular internal diameter at end-systole; LVPW-s, left ventricular posterior wall thickness at end-systole; EF\%, ejection fraction; FS\%, fractional shortening

hypoxia/reperfusion induced cardiomyocyte apoptosis through inhibition of mitochondria-related cell death pathway, ROS accumulation, and diastolic calcium abnormality. ${ }^{23}$ Structurally, Nogo-C shares the transmembrane domain and C-terminal domain with Nogo-A, whereas missing the N-terminal domain contained by Nogo-A protein. ${ }^{26,27} \mathrm{So}$, it is possible that the common domains shared by the two Nogo proteins contribute to the apoptotic effects, the same as the inhibitory effect of regeneration in nervous system. ${ }^{18}$ However, the exact molecular mechanisms of Nogo-C-induced cardiomyocyte apoptosis need further exploration.

Our above results on Nogo-C and others' previous studies on Nogo-A indicate that the increased Nogo proteins during $\mathrm{Ml}$ or ischemic heart diseases may contribute to cardiac dysfunction through regulating cardiomyocyte apoptosis. To better understand the pathophysiological significance of Nogo- $\mathrm{C}$ in the heart, we generated the Nogo- $\mathrm{C}$ knockout mouse model, the first Nogo-C knockout model to our knowledge. Our in vivo functional study in Nogo-C null mice provides solid evidence supporting our hypothesis, that depletion of Nogo-C protected cardiomyocyte apoptosis in MI heart, largely decreased IR, and most importantly, preserved cardiac function after $\mathrm{Ml}$ injury, suggesting that Nogo-C may serve as a therapeutic target for treatment of $\mathrm{MI}$ or ischemia-related cardiac diseases. Our present study of the protective effect of Nogo-C knockout in the heart is in general agreement with Nogo-A knockout mouse model. ${ }^{23}$ Although Nogo-C knockout protected the heart from Ml damage, it showed no cardiac phenotype at basal level, suggesting that Nogo-C is either dispensable for normal functions or there are redundant pathways in the heart. Our preliminary data found that mRNA levels of other two Nogo family members, Nogo-A and Nogo-B, were increased in Nogo-C KO mouse hearts, suggesting that elevated Nogo proteins may at least partially provide compensatory function in Nogo-C KO hearts. In this respect, the protective effect of Nogo-C knockout independent of the compensatorily increased Nogo-A suggests that Nogo-C and Nogo-A may differentially regulate cardiomyocyte apoptosis. Indeed, Nogo-A induced cardiomyocyte apoptosis through a mitochondria dependent pathway, ${ }^{23}$ whereas Nogo-C had no effect on mitochondria (data not shown).
MicroRNAs (miRNAs) have a crucial role in the pathogenesis of various cardiac diseases including $\mathrm{Ml}$, post-infarct fibrosis, and heart failure. ${ }^{28-33}$ In mouse and human hearts, the expression profile of miRNAs in the border zone of infarct region was altered after $\mathrm{Ml}$ and during the subsequent cardiac fibrosis. $^{32}$ Downregulation of miRNAs especially miR-29 family de-repressed its target genes, which encode fibrosisrelated proteins, causing post-infarct fibrosis and cardiac dysfunction. ${ }^{34}$ In the current study, we predicted, according to the $3^{\prime} U T R$ region of Nogo-C, that Nogo-C is a target gene of $\mathrm{miR}-182$. In vitro expression of miR-182 reduced Nogo-C protein level and Nogo-C 3 '-UTR luciferase reporter activity, confirming our prediction that miR-182 negatively regulates Nogo-C expression. Moreover, the expression of miR-182 was decreased in the border zone of infarct heart and in hypoxic cardiomyocytes, suggesting that miR-182 may be involved in the pathological processes of $\mathrm{Ml}$ and ischemic heart diseases. MiR-182 has been reported to participate in the regulation of multiple physiological and pathological processes including retinal development and degeneration, ${ }^{35,36}$ cancers, ${ }^{37-40}$ inflammation, ${ }^{41,42}$ and cardiac hypertrophy. ${ }^{43}$ Controversially, miR-182 showed tumor-suppressive function in some cancers such as glioblastoma, ${ }^{44}$ whereas displayed ongogenic function in other cancers such as lung adenocarcinoma. ${ }^{45}$ In our study, we found that miR-182 protected cardiomyocytes from Nogo-C and hypoxia-induced apoptosis. It is unclear why miR-182 functions differentially on cell growth or apoptosis in cancers or in the heart, tissue specific effect might be a possible reason for this discrepancy. However, how miR-182 is regulated during $\mathrm{Ml}$ and ischemic heart is not yet clear and merits further investigation.

In summary, our present study found that Nogo-C protein was upregulated in MI heart and hypoxic cardiomyocytes, the increased Nogo-C induced cardiomyocyte apoptosis and silencing Nogo-C protected cardiomyocytes from hypoxiainduced apoptosis. Knockout of Nogo-C protected the heart from $\mathrm{Ml}$ injury and preserved cardiac function. Furthermore, we identified that miR-182 is a negative regulator of Nogo-C, and downregulation of miR-182 may contribute to the increased Nogo-C and cardiac dysfunction during MI. Our findings reveal a critical role of Nogo-C in the heart, and thus shed new light on the development of therapeutic targets for ischemia-related cardiac diseases.

\section{Materials and Methods}

Mouse MI model. All procedures of animal handling were approved by the Institutional Animal Care and Use Committee of Peking University Health Science Center. Mouse MI model was established with C57BL/6 male mice at age of 8-12week as previously described. ${ }^{46}$ Mice were anesthetized with intraperitoneal injection of pentobarbital sodium ( $60 \mathrm{mg} / \mathrm{kg}$ ). The fourth intercostals space over the left chest was exposed and the heart was rapidly squeezed out of the thoracic space, the LAD below the tip of the left auricle was tied with a 6 sterile silk suture. The sham subjects underwent same operation except that the LAD was not ligated. Twenty-four hours after the operation, echocardiography was performed with a Vevo 710 RMV-707B (VisualSonics, Toronto, Ontario, Canada), and hearts were collected and prepared for experiments.

Generation of Nogo-C knockout mouse model. Nogo-C knockout mice (Nogo- $\mathrm{C}^{-1-}$ ) were generated by TALEN technique in C57BL/6 background. Briefly, 8 basepairs of exon 1c of Nogo gene, the specific exon for Nogo-C, were chopped to induce a frame-shift mutation, resulting in a truncated protein, which 
a
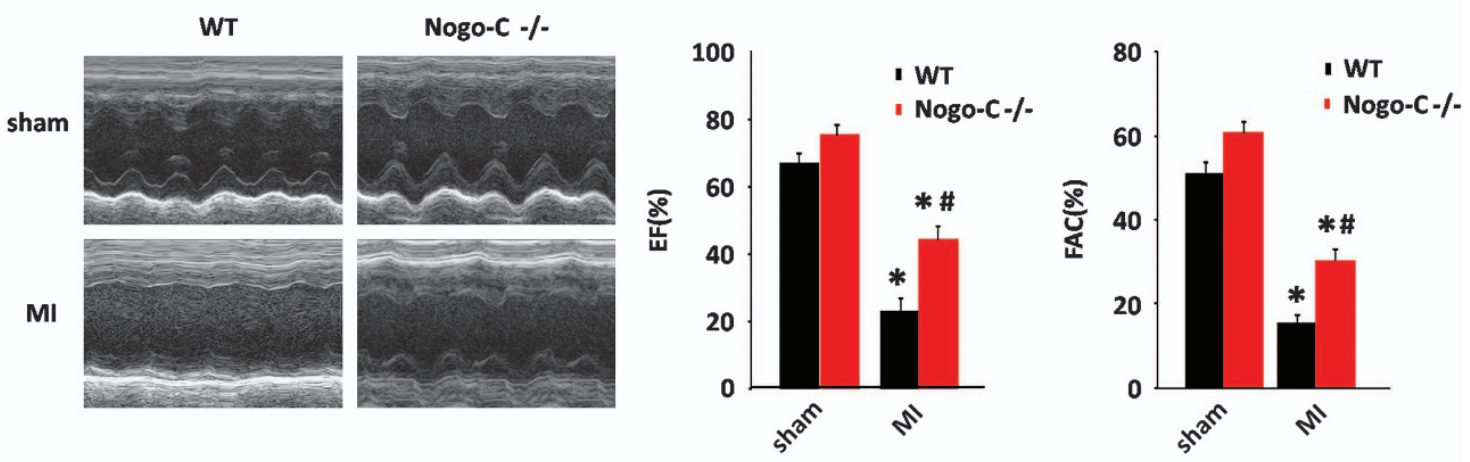

b
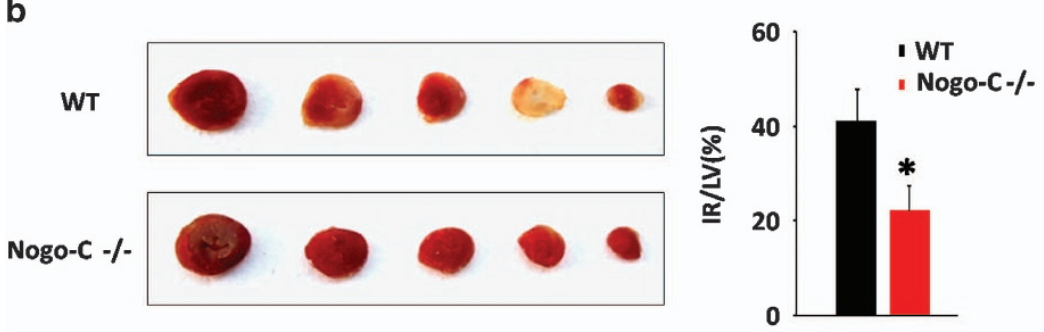

C

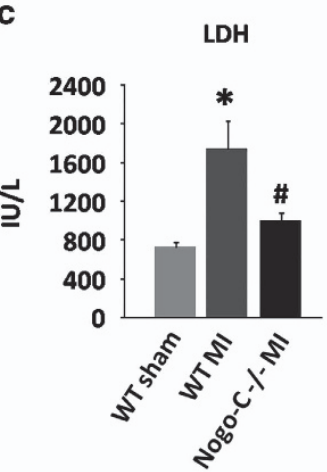

d
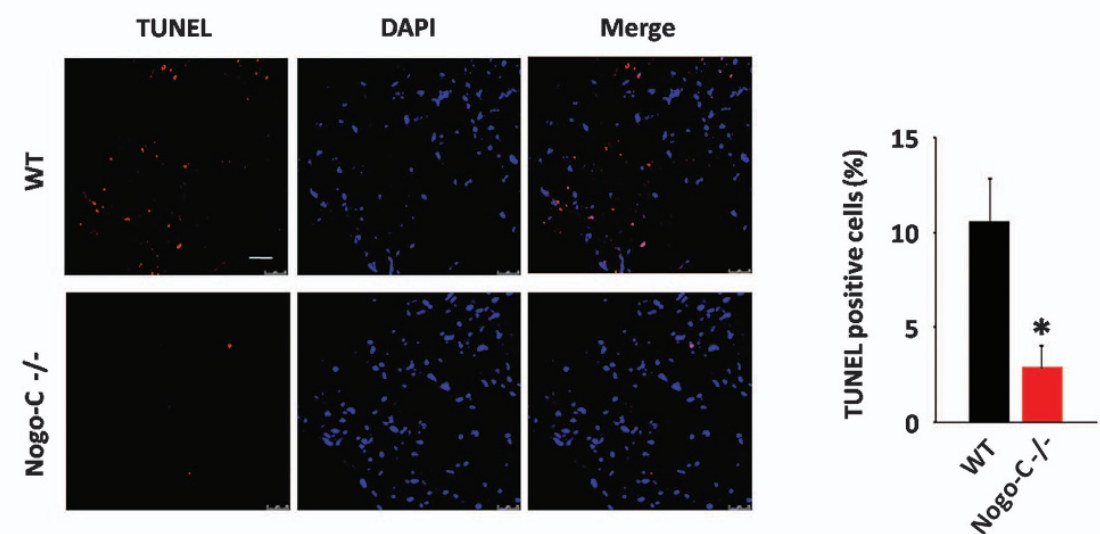

Figure 4 Nogo-C knockout protects mouse heart from MI injury. (a) Typical example of M-mode echocardiograms of wildtype and Nogo- $C^{-1-}$ mouse hearts with (MI) or without (sham) LAD ligation for $24 \mathrm{~h}$ (left), and average data of $\mathrm{EF}$ (middle) and FAC (right). $n=6$ mice for each group. ${ }^{*} P<0.05$ versus sham wildtype mice; ${ }^{\#} P<0.05$ versus $\mathrm{MI}$ wildtype mice. (b) Representative triphenyltetrazolium chloride (TTC) staining images of sequential heart sections (left), and the average data of TTC infarction size (right) of WT and Nogo- $C^{-1-}$ mice after LAD ligation for $24 \mathrm{~h} . n=4$ mice, ${ }^{\star} P<0.05$ versus wildtype mice. (c) Serum LDH activities of wildtype and Nogo- $C^{-1-}$ mice with or without LAD ligation for 24 h. $n=5-11$ mice. ${ }^{*} P<0.05$ versus WT sham; ${ }^{\#} P<0.05$ versus WT MI. (d) TUNEL staining showing apoptotic cells in the border zone of MI from WT and Nogo- $\mathrm{C}^{-/-}$mouse hearts $24 \mathrm{~h}$ after LAD ligation (left), and the average data (right). $n=4$ mice. Scale bar $=25 \mu \mathrm{m} .{ }^{\star} P<0.05$ versus wildtype mice

may be subject to non-sense mediated decay. Knockout of Nogo-C was confirmed by western blot and gene sequencing.

Immunohistochemical staining assay. Heart samples were fixed with $4 \%$ paraformaldehyde and imbedded with paraffin wax. The sections were perfused in $3 \% \mathrm{H}_{2} \mathrm{O}_{2}$ to clear endogenous peroxidase and microwaved in sodium citrate buffer $(1 \mathrm{mM}, \mathrm{pH} 6)$. The slides were blocked in $1 \% \mathrm{BSA}$ for $30 \mathrm{~min}$ at $37^{\circ} \mathrm{C}$ and were then probed with anti-Nogo-C antibody $(1: 100)$ overnight at $4{ }^{\circ} \mathrm{C}$ and a horseradish peroxidase conjugated secondary antibody at $37^{\circ} \mathrm{C}$ for $30 \mathrm{~min}$.

Isolation and culture of rat neonatal cardiomyocytes. Rat neonatal cardiomyocytes were isolated and cultured as described previously. ${ }^{22}$ Briefly, ventricles of Sprague-Dawley rats postnatal 1 to 2 days were digested in HBSS solution (KCl $0.4 \mathrm{gll}, \mathrm{KH}_{2} \mathrm{PO}_{4} 0.06 \mathrm{gll}, \mathrm{NaHCO}_{3} 0.35 \mathrm{gll}, \mathrm{NaCl}: 8 \mathrm{gll}, \mathrm{Na}_{2} \mathrm{HPO}_{4} 12 \mathrm{H}_{2} \mathrm{O}$ $0.12 \mathrm{~g} / \mathrm{l}$, glucose $1 \mathrm{gl}, \mathrm{pH} 7.4$ ) containing $0.1 \%$ trypsin (Invitrogen, Carlsbad, CA, USA) and $0.05 \%$ type II collagenase (Worthington, Lakewood, CO, USA). Cells were pre-plated for $2 \mathrm{~h}$ and the supernatant containing purified cardiomyocytes was collected and re-plated and cultured for another $48-72 \mathrm{~h}$ before transfecting adenovirus.

Construction of adenovirus. The full-length CDNA of Nogo-C was amplified by PCR with primers: 5'-CACCATGGACG GACAGAAGAAACA-3' (forward) and 5'-TCAATCTGCTTTGCGCTTCAATCC-3' (reverse), the amplified product was then inserted into pENTR/TEV/D-TOPO vector (Invitrogen). Newly constructed product was recombined with pAd/CMV/N5-DEST vector (Invitrogen). Target sequence for Nogo-C-shRNA was 5'-GGCAGATCGTGGCAAGAAA-3'. The Nogo-C-shRNA sequence was inserted into $\mathrm{pENTR}^{\mathrm{TM}} / \mathrm{U} 6$ vector and then recombined with $\mathrm{pBLOCK}-\mathrm{T}^{\mathrm{TM}}{ }_{6}$-DEST vector. Adenovirus was produced with Adenoviral Expression System (Invitrogen) and purified using Vivapure AdenoPACK20RT Kit (Sartorius, Göttingen, Germany).

Immunoblotting. Protein was extracted from mouse myocardium or isolated cardiomyocytes with Roth lysis buffer (HEPES 50 mM, NaCl 150 mM, EDTA 5 mM, 


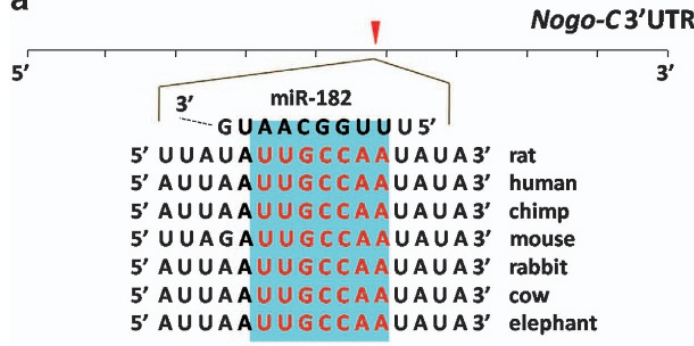

d

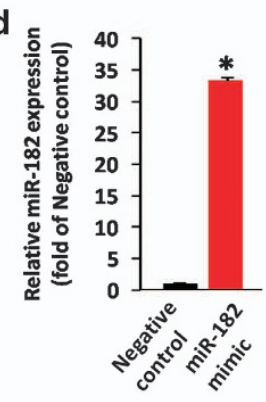

e

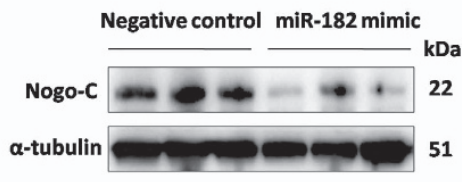

b
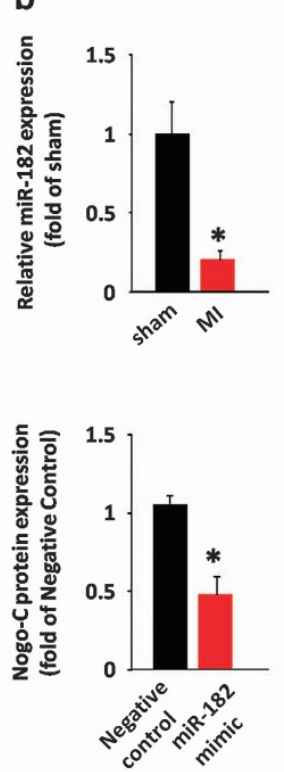

C

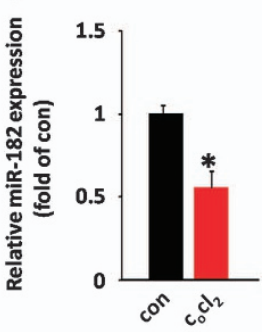

f

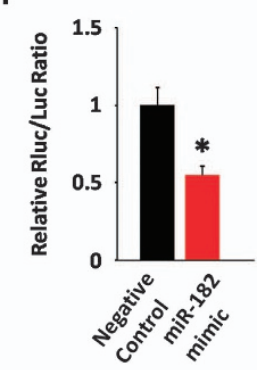

g

9 Con

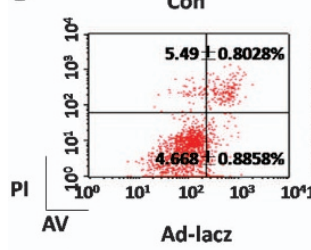

$\mathrm{CoCl}_{2}$

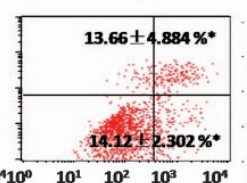

Ad-Nogo-C

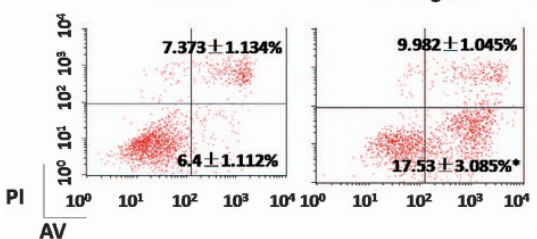

$\mathrm{CoCl}_{2}+\mathrm{miR}-182$ mimic

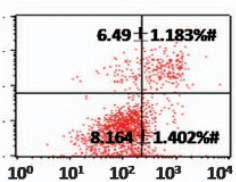

Ad-Nogo-C+miR-182 mimic

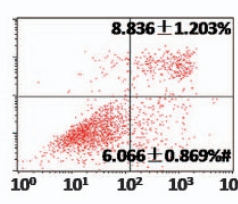

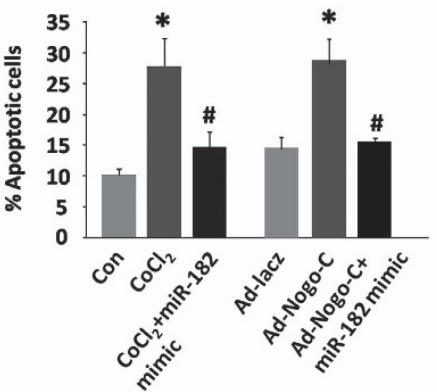

Figure 5 miR-182 regulates Nogo-C expression. (a) Analysis of Nogo-C 3'UTR region predicting miR-182 as a regulator. PCR data showing miR-182 expression in MI hearts (b) or $\mathrm{CoCl}_{2}$-treated cardiomyocytes (c). (d) Expression of miR-182 in control and miR-182 mimic transfected rat neonatal cardiomyocytes. (e) Western blot (left) and average data (right) showing the Nogo-C protein levels in control and miR-182 overexpressing rat neonatal cardiomyocytes. (f) Luciferase reporter activity of rat Nogo-C $3^{\prime}$ UTR in cells transfected with miR-182 mimic or scrambled miRNA mimic for $48 \mathrm{~h}$. (g) Flow cytometry analysis showing apoptotic rates of rat neonatal cardiomyocytes transfected with or without miR-182 mimic in response to $\mathrm{CoCl}_{2}$ stimulation $(600 \mu \mathrm{M})$ or Nogo-C overexpression. $n=6$ independent experiments. ${ }^{*} P<0.05$ versus control cells. ${ }^{\#} P<0.05$ versus $\mathrm{CoCl}_{2}$ or Nogo-C overexpressing cells

EGTA $5 \mathrm{mM}, \mathrm{NaF} 20 \mathrm{mM}$, Triton X-100 1\%, pH 7.4) supplemented with $100 \mathrm{X}$ protease inhibitor cocktail (Sigma, Santa Clara, CA, USA). A total of 40-100 ug protein was then separated by SDS-PAGE, and transferred to PVDF membranes. Membranes were probed with indicated primary antibodies (Nogo-C or $\alpha$-tubulin), and then incubated with the secondary antibodies (horseradish peroxidaseconjugated anti-rabbit IgG from ZSGB-BIO, Beijing, China). Immunoblots were evaluated using the Chemi Doc XRS+instrument (Bio-RAD, Hercules, CA, USA).

MiRNA assay. MicroRNA assay was detected with stem loop primers purchased from Ribobio (Guangzhou RiboBio, Guangzhou, China). U6 small nucleolar RNA (Guangzhou RiboBio) was used for the normalization. The sequence of rno-mir-182 was 5'-ACGCGGGUCUAGCUGCCGGAGGCCUCCCACCGUUUUU GGCAAUGGUAGAACUCACACCGGUA-3'. Rno-mir-182 mimics (Guangzhou RiboBio) were transfected to rat neonatal cardiomyocytes with Lipofectamine 3000 (Invitrogen).

Dual luciferase reporter assay. Nogo-C $3^{\prime}$-UTR sequences were amplified by PCR with primers: 5'-GCGCTCGAGAAAAGC CCCAAACAGAAGT-3' (forward) and $5^{\prime}$-AATGCGGCCGCAAACACTAAACACAAACAT-3' (reverse). PCR products were then constructed into pmiR-RB-REPORT plasmid (Guangzhou RiboBio) containing two reporters, a renilla luciferase (hRluc) for expression evaluation, and a firefly luciferase (hluc) as an internal control for amount correlation. Hela cells were co-transfected with $100 \mathrm{ng}$ Nogo-C $3^{\prime}$ UTR reporter plasmid and $2 \mu \mathrm{g}$ rno-miR-182 mimic or empty vector. Cells were collected $48 \mathrm{~h}$ after transfection and analyzed using a Dual Luciferase Reporter Assay System (Promega, Madison, WI, USA). Luciferase activity was examined by Veritas luminometer (Turner BioSystems, Sunnyvale, CA, USA). Renilla luciferase activity of each sample was normalized to firefly luciferase activity.

Cell death assay by TUNEL staining and flow cytometry. Cell apoptosis was determined by TUNEL (terminal deoxyribonucleotidyl transferase (TDT)-mediated dUTP-digoxigenin nick end labeling) assay using In situ cell death detection kit (Roche, Indianapolis, IN, USA), or by fluorescein isothiocyanate-conjugated annexin V/propidium iodide double staining flow cytometry using Annexin-V-FITC Apoptosis Detection Kit (Dojindo Laboratories, Kumamoto-ken, Japan). 
Transmission electron microscopy analyses. Hearts were fixed with $2 \%$ glutaraldehydein sodium cacodylate buffer $(0.1 \mathrm{M}, \mathrm{pH} 7.2)$ at $4{ }^{\circ} \mathrm{C}$ overnight and post-fixed for $1 \mathrm{~h}$ in $1 \%$ osmiumtetroxide. Digital images were acquired by a JEM-1230 High Contrast Transmission Electron Microscope and Soft Imaging system (JEOL, Tokyo, Japan).

TTC staining. Mice were killed with anesthetic $24 \mathrm{~h}$ after Ml operation. Hearts were frozen at $-20^{\circ} \mathrm{C}$ for $20 \mathrm{~min}$, and then were sectioned into $5 \mathrm{~mm}$ thick slices. Five continuous slices from apex to the occlusion site were incubated in triphenyltetrazolium chloride (TTC) at $37^{\circ} \mathrm{C}$ for $5 \mathrm{~min}$. After fixation with $4 \%$ paraformaldehyde overnight, each slice was weighed $(w)$ and photographed with a digital camera. Infarct areas were indicated as the area not stained by TTC. The IR and left ventricular size $(\mathrm{LV})$ were evaluated by Photoshop. Percentage of infarct area was calculated as $I R / L V=\left(\sum \mathbb{R}_{\text {(per slice) }} \times w_{\text {(per slice) }}\right) /\left(\sum L V_{(\text {per slice) }} \times w_{(\text {per slice) }}\right)$.

LDH assay. One milliliter vein blood was collected from right ventricular with a syringe and then centrifuged at 3000 r.p.m. for $15 \mathrm{~min}, 4^{\circ} \mathrm{C}$. Serum samples were sent to clinical laboratory of the Third Hospital at Peking University for analyses of LDH levels.

Statistical analysis. Data are presented as mean \pm S.E.M. Statistical significance of differences between groups was analyzed by unpaired $t$-test or one-way ANOVA followed by SNK (Student-Newman-Keuls) when more than two groups were compared. $P<0.05$ was considered statistically significant.

\section{Conflict of Interest}

The authors declare no conflict of interest.

Acknowledgements. This work was supported by grants from the National Key Basic Research Program of China (2013CB531203 and 2014CB542206) and National Science Foundation of China (31371350 and 81670233).

1. Finegold JA, Asaria P, Francis DP. Mortality from ischaemic heart disease by country, region, and age: statistics from World Health Organisation and United Nations. Int J Cardiol 2013; 168: 934-945.

2. Katz AR. Noncommunicable diseases: global health priority or market opportunity? An illustration of the World Health Organization at its worst and at its best. Int $\mathrm{J}$ Health Serv 2013; 43: 437-458.

3. Kloner RA. New observations regarding post-ischemia/reperfusion myocardial swelling J Am Coll Cardiol 2015; 65: 324-326.

4. Sugamura K, Keaney JF Jr. Reactive oxygen species in cardiovascular disease. Free Radic Biol Med 2011; 51: 978-992.

5. Waza AA, Andrabi K, Hussain MU. Protein kinase $C(P K C)$ mediated interaction between conexin43 (Cx43) and $\mathrm{K}(+)(\mathrm{ATP})$ channel subunit (Kir6.1) in cardiomyocyte mitochondria: Implications in cytoprotection against hypoxia induced cell apoptosis. Cell Signal 2014; 26: 1909-1917.

6. Yokota T, Wang Y. p38 MAP kinases in the heart. Gene 2016; 575: 369-376.

7. Senga $Y$, Satomi $Y$, Fukuda M, Misugi K. A case of renal cell carcinoma in childhood. Nihon Hinyokika Gakkai Zasshi 1985; 76: 1573-1579.

8. Chen MS, Huber AB, van der Haar ME, Frank M, Schnell L, Spillmann AA et al. Nogo-A is a myelin-associated neurite outgrowth inhibitor and an antigen for monoclonal antibody IN-1. Nature 2000; 403: 434-439.

9. Brosamle C, Huber AB, Fiedler M, Skerra A, Schwab ME. Regeneration of lesioned corticospinal tract fibers in the adult rat induced by a recombinant, humanized IN-1 antibody fragment. J Neurosci 2000; 20: 8061-8068.

10. Petrinovic MM, Duncan CS, Bourikas D, Weinman O, Montani L, Schroeter A et al. Neuronal Nogo-A regulates neurite fasciculation, branching and extension in the developing nervous system. Development 2010; 137: 2539-2550.

11. Walchli T, Pernet V, Weinmann O, Shiu JY, Guzik-Kornacka A, Decrey G et al. Nogo-A is a negative regulator of CNS angiogenesis. Proc Natl Acad Sciences USA 2013; 110: E1943-E1952.

12. Huber AB, Weinmann O, Brosamle $C$, Oertle T, Schwab ME. Patterns of Nogo mRNA and protein expression in the developing and adult rat and after CNS lesions. J Neurosci 2002; 22: 3553-3567.

13. Eckharter C, Junker N, Winter L, Fischer I, Fogli B, Kistner S et al. Schwann cell expressed Nogo-B modulates axonal branching of adult sensory neurons through the Nogo-B receptor NgBR. Front Cell Neurosci 2015; 9: 454
14. Di Lorenzo A, Manes TD, Davalos A, Wright PL, Sessa WC. Endothelial reticulon-4B (Nogo-B) regulates ICAM-1-mediated leukocyte transmigration and acute inflammation. Blood 2011; 117: 2284-2295.

15. Sutendra G, Dromparis P, Wright P, Bonnet S, Haromy A, Hao Z et al. The role of Nogo and the mitochondria-endoplasmic reticulum unit in pulmonary hypertension. Sci Transl Med 2011; 3: 88ra55.

16. Marklund N, Morales D, Clausen F, Hanell A, Kiwanuka O, Pitkanen A et al. Functiona outcome is impaired following traumatic brain injury in aging Nogo-A/B-deficient mice. Neuroscience 2009; 163: 540-551.

17. Geisler JG, Stubbs LJ, Wasserman WW, Mucenski ML. Molecular cloning of a novel mouse gene with predominant muscle and neural expression. Mamm Genome 1998; 9: 274-282.

18. Kim JE, Bonilla IE, Qiu D, Strittmatter SM. Nogo-C is sufficient to delay nerve regeneration. Mol Cell Neurosci 2003; 23: 451-459.

19. Chen $Y$, Tang $X$, Zhang $X$, Zhuang L. New mutations of Nogo-C in hepatocellular carcinoma. Mol Biol Rep 2009; 36: 377-380.

20. Liu X, Cui SJ, Zhu SJ, Geng DC, Yu L. Nogo-C contributes to HCC tumorigenesis via suppressing cell growth and its interactome analysis with comparative proteomics research. Int J Clin Exp Pathol 2014; 7: 2044-2055.

21. Chen $Y$, Tang X, Cao X, Chen H, Zhang X. Human Nogo-C overexpression induces HEK293 cell apoptosis via a mechanism that involves JNK-c-Jun pathway. Biochem Biophys Res Commun 2006; 348: 923-928.

22. Hao CQ, Zhou Y, Wang JP, Peng MJ, Xie YM, Kang WZ et al. Role of NogoA in the regulation of hepatocellular carcinoma SMMC7721 cell apoptosis. Mol Med Rep 2014; 9: 1743-1748

23. Sarkey JP, Chu M, McShane M, Bovo E, Ait Mou Y, Zima AV et al. Nogo-A knockdown inhibits hypoxia/reoxygenation-induced activation of mitochondrial-dependent apoptosis in cardiomyocytes. J Mol Cell Cardiol 2011; 50: 1044-1055.

24. Palojoki E, Saraste A, Eriksson A, Pulkki K, Kallajoki M, Voipio-Pulkki LM et al. Cardiomyocyte apoptosis and ventricular remodeling after myocardial infarction in rats. Am J Physiol Heart Circ Physiol 2001; 280: H2726-H2731.

25. Kajstura J, Cheng W, Reiss K, Clark WA, Sonnenblick EH, Krajewski S et al. Apoptotic and necrotic myocyte cell deaths are independent contributing variables of infarct size in rats. Lab Invest 1996; 74: 86-107.

26. Goldberg JL, Barres BA. Nogo in nerve regeneration. Nature 2000; 403: 369-370.

27. GrandPre T, Nakamura F, Vartanian T, Strittmatter SM. Identification of the Nogo inhibitor of axon regeneration as a Reticulon protein. Nature 2000; 403: 439-444.

28. Weiss JB, Eisenhardt SU, Stark GB, Bode C, Moser M, Grundmann S. MicroRNAs in ischemia-reperfusion injury. Am J Cardiovasc Dis 2012; 2: 237-247.

29. Nagpal V, Rai R, Place AT, Murphy SB, Verma SK, Ghosh AK et al. MiR-125b is critical for fibroblast-to-myofibroblast transition and cardiac fibrosis. Circulation 2016; 133 291-301.

30. Li Q, Xie J, Wang B, Li R, Bai J, Ding L et al. Overexpression of microRNA-99a attenuates cardiac hypertrophy. PLoS One 2016; 11: e0148480.

31. el Azzouzi H, Leptidis S, Dirkx E, Hoeks J, van Bree B, Brand K et al. The hypoxia-inducible microRNA cluster miR-199a approximately 214 targets myocardial PPARdelta and impairs mitochondrial fatty acid oxidation. Cell Metab 2013; 18: 341-354.

32. Hullinger TG, Montgomery RL, Seto AG, Dickinson BA, Semus HM, Lynch JM et al. Inhibition of miR-15 protects against cardiac ischemic injury. Circ Res 2012; 110: $71-81$.

33. Wong LL, Wang J, Liew OW, Richards AM, Chen YT. MicroRNA and heart failure. Int J Mol Sci 2016; 17: 502.

34. Li M, Wang N, Zhang J, He HP, Gong HQ, Zhang R et al. MicroRNA-29a-3p attenuates ET-1-induced hypertrophic responses in H9c2 cardiomyocytes. Gene 2016; 585 : 44-50.

35. Lumayag S, Haldin CE, Corbett NJ, Wahlin KJ, Cowan C, Turturro S et al. Inactivation of the microRNA-183/96/182 cluster results in syndromic retinal degeneration. Proc Natl Acad SCi USA 2013; 110: E507-E516.

36. Zhu Q, Sun W, Okano K, Chen Y, Zhang N, Maeda T et al. Sponge transgenic mouse model reveals important roles for the microRNA-183 (miR-183)/96/182 cluster in postmitotic photoreceptors of the retina. J Biol Chem 2011; 286: 31749-31760.

37. Segura MF, Hanniford D, Menendez S, Reavie L, Zou X, Alvarez-Diaz S et al. Aberrant miR-182 expression promotes melanoma metastasis by repressing FOXO3 and microphthalmia-associated transcription factor. Proc Natl Acad Sci USA 2009; 106 : 1814-1819.

38. Chen Q, Yang L, Xiao Y, Zhu J, Li Z. Circulating microRNA-182 in plasma and its potential diagnostic and prognostic value for pancreatic cancer. Med Oncol 2014; 31: 225.

39. McMillen BD, Aponte MM, Liu Z, Helenowski IB, Scholtens DM, Buttin BM et al. Expression analysis of MIR182 and its associated target genes in advanced ovarian carcinoma. Mod Pathol 2012; 25: 1644-1653.

40. Du C, Weng X, Hu W, Lv Z, Xiao H, Ding $\mathrm{C}$ et al. Hypoxia-inducible MiR-182 promotes angiogenesis by targeting RASA1 in hepatocellular carcinoma. J Exp Clin Cancer Res 2015; 34: 67

41. Kelada S, Sethupathy P, Okoye IS, Kistasis E, Czieso S, White SD et al. miR-182 and miR-10a are key regulators of Treg specialisation and stability during Schistosome and Leishmania-associated inflammation. PLoS Pathog 2013; 9: e1003451. 
42. Pucella JN, Yen WF, Kim MV, van der Veeken J, Luo CT, Socci ND et al. miR-182 is largely dispensable for adaptive immunity: lack of correlation between expression and function J Immunol 2015; 194: 2635-2642.

43. Li N, Hwangbo C, Jaba IM, Zhang J, Papangeli I, Han J et al. miR-182 modulates myocardia hypertrophic response induced by angiogenesis in heart. Sci Rep 2016; 6: 21228.

44. Kouri FM, Hurley LA, Daniel WL, Day ES, Hua Y, Hao L et al. miR-182 integrates apoptosis, growth, and differentiation programs in glioblastoma. Genes Dev 2015; 29 : 732-745.

45. Wang M, Wang Y, Zang W, Wang H, Chu H, Li P et al. Downregulation of microRNA-182 inhibits cell growth and invasion by targeting programmed cell death 4 in human lung adenocarcinoma cells. Tumour Biol 2014; 35: 39-46.

46. Gao E, Lei YH, Shang X, Huang ZM, Zuo L, Boucher M et al. A novel and efficient model of coronary artery ligation and myocardial infarction in the mouse. Circ Res 2010; 107: 1445-1453. cc (i) Cell Death and Disease is an open-access journal published by Nature Publishing Group. This work is licensed under a Creative Commons Attribution 4.0 International License. The images or other third party material in this article are included in the article's Creative Commons license, unless indicated otherwise in the credit line; if the material is not included under the Creative Commons license, users will need to obtain permission from the license holder to reproduce the material. To view a copy of this license, visit http://creativecommons.org/licenses/by/4.0/

(C) The Author(s) 2016 